\title{
Line-shape modeling in microwave spectroscopy of the positronium $n=2$ fine-structure intervals
}

\author{
L. A. Akopyan $\odot,{ }^{1, *}$ T. J. Babij, ${ }^{2}$ K. Lakhmanskiy $\odot,{ }^{1}$ D. B. Cassidy $\odot,{ }^{2}$ and A. Matveev $\oplus^{1}$ \\ ${ }^{1}$ Russian Quantum Center, Skolkovo, Moscow 143025, Russia \\ ${ }^{2}$ Department of Physics and Astronomy, University College London, Gower Street, London WC1E 6BT, United Kingdom
}

(Received 19 October 2021; accepted 2 December 2021; published 16 December 2021)

\begin{abstract}
We report numerical simulations of positronium experiments designed to measure the $n=2$ fine-structure intervals. The simulations include all possible interference effects between all 20 states in the $n=1$ and laser-excited $n=2$ manifolds as well as representations of the electric and magnetic fields present in the waveguides used in the experiments. We find that $\mathrm{rf}$ wave reflection from the vacuum chamber walls is a possible explanation of previously observed line-shape distortions and shifts. We also characterized several systematic effects, including those arising from quantum interference, that are likely to be significant for future measurements.
\end{abstract}

DOI: 10.1103/PhysRevA.104.062810

\section{INTRODUCTION}

Positronium (Ps) is an exotic hydrogenic atom composed of an electron bound to its antiparticle, the positron [1]. Being composed of two low-mass leptons, strong and weak interactions can be entirely neglected and there are no nuclear structure complications, meaning that Ps is in effect fully described by bound-state QED, and precision Ps spectroscopy can be used to test this theory to a high degree [2]. Since Ps is a pure QED system, such tests are naturally sensitive to nonQED effects, such as new particles, forces, or interactions not included in the QED theory, but only if they can be performed with a precision commensurate with the relevant QED theory. Unfortunately experimental precision in Ps measurements [3] is far behind the theoretical situation [4].

Some recent measurements of the Ps $n=2$ fine structure have been conducted with the aim of closing the gap between theory and experiment $[5,6]$. Previous measurements of the $2{ }^{3} S_{1} \rightarrow 2{ }^{3} P_{J}$ intervals (where $J=0,1,2$, and the transitions are henceforth denoted as $v_{J}$ ) have been conducted decades ago, achieving uncertainties on the order of several $\mathrm{MHz}$ [7-9], while the calculations have estimated uncertainties of $80 \mathrm{kHz}$ [4]. By using new positron trapping [10] and Ps production techniques [3], the new measurements were able to improve the measurement uncertainties to sub- $\mathrm{MHz}$ levels. However, it was found that the measured $v_{0}$ transition interval disagreed with the QED calculation by more than four standard deviations [5]. Moreover, the $v_{1}$ and $v_{2}$ measurements exhibited unexplained asymmetric line shapes [6] which prevented a precise determination of the corresponding intervals.

It is well known that line centers of resonances can be affected by the presence of nearby off-resonant pathways and that shifts and asymmetric line shapes can result (e.g., Refs. [11,12]). Indeed, these types of quantum interference (QI) effects have had to be taken into account in several highprecision measurements (e.g., Refs. $[13,14])$. In the recent Ps fine-structure experiments a simplified form of this effect was

\footnotetext{
*1.akopyan@rqc.ru
}

considered [6] and found not to contribute at the level of the observed discrepancies. Here we present the results of a more complete density-matrix model using numerical integration of a master equation in the Lindblad form [15]. This model includes all Ps states in the $n=1$ and $n=2$ manifolds, and provides a full description of all relevant QI effects in the Ps measurements.

In addition, the model included explicit representations of the electric and magnetic fields inside the waveguides used in the experimental apparatus, including contributions arising from reflections from the surrounding vacuum chambers. These fields were modeled numerically using finite element methods (FEM), and made it possible to perform complete simulations of the experimental conditions, and thus to fully characterize the extent to which QI effects and nonideal electromagnetic fields may have affected the measurements. We find that, as expected, QI effects are indeed present, but are too small to account for the anomalous observations. However, some frequency-dependent microwave radiation reflection effects have been identified that may (at least qualitatively) explain the observations.

The article is arranged in the following way. Section II contains a description of the experimental setup and explains in broad terms how it is modeled, including the particle trajectories, signal extraction, and line-shape production for ideal microwave fields. Section III describes the quantum dynamics of the system using the Lindblad formalism, which naturally includes all QI effects. In Sec. IV realistic microwave fields are modeled using finite element methods, and are added to the simulations along with the density-matrix treatment of Sec. III. Additional (smaller) systematic effects are considered in Sec. V for completeness, and we draw some conclusions from this work in Sec. VI.

\section{MODEL OF EXPERIMENT}

Previously, simulations were performed that were designed to represent the experimental arrangements used in recent measurements of the Ps fine structure [5,6]. However, not all 


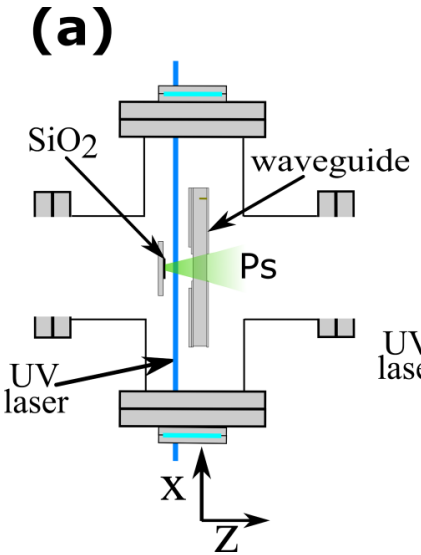

(b)

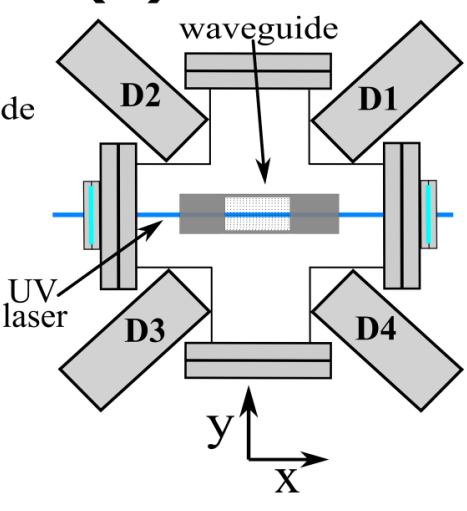

FIG. 1. Experimental configuration showing (a) the Ps formation relative to the waveguide location and (b) the positions of four $\gamma$-ray detectors around the vacuum chamber, for a WR-112 waveguide.

aspects of the experiments were simulated; for example, in the experiments Ps atoms in the $2{ }^{3} S_{1}$ level were generated via a single-photon excitation process in a time varying electric field [16]. Here we do not consider the initial-state preparation, and assume instead that $2^{3} S_{1}$ atoms appear at an initial time $t=0$ at a point in space $\vec{r}_{0}=(0,0,0)$. This initial source spot represents the point where the positron beam is implanted into a mesoporous silica target, from which the positrons are emitted [17]. The actual positron beam has a Gaussian profile with a full width at half maximum (FWHM) of $3 \mathrm{~mm}$ [18]. The finite Ps source size does not affect the simulations in any significant way and has been neglected. The Ps formation process is also not explicitly simulated, but the resulting Ps velocity distributions have been measured and are included, as discussed below.

\section{A. Geometry and particle trajectories}

The experimental geometry is indicated in Fig. 1. Ps atoms produced from a porous silica film [17] are emitted into an excitation region where a laser drives $1^{3} S_{1} \rightarrow 2^{3} P_{J}$ transitions in a strong electric field. The electric fields perturb the excited state via the Stark effect, allowing atoms to evolve to the $2{ }^{3} S_{1}$ level after it is turned off [16]. Excited-state atoms subsequently pass through a grid in the side of a rectangular waveguide. This can be one of three different types, depending on which transition is to be studied (see Table I): a WR-112 waveguide, used for $2{ }^{3} S_{1} \rightarrow 2{ }^{3} P_{2}$ (i.e., $v_{2}$ ) transitions, is shown in Fig. 1.

TABLE I. Specifications of the three waveguides used in the experiment and simulations. The dimensions listed refer to the distances between the inner walls in the $y, z$, and $x$ dimensions (see Fig. 1). The cutoff frequencies refer to the lowest-order $\mathrm{TE}_{10}$ modes.

\begin{tabular}{lccc}
\hline \hline Waveguide & Dimensions $(\mathrm{mm})$ & Range $(\mathrm{GHz})$ & Cutoff $(\mathrm{GHz})$ \\
\hline WR-51(v $\left.v_{0}\right)$ & $12.95 \times 6.48 \times 160$ & $15-22$ & 11.58 \\
WR-75 $\left(v_{1}\right)$ & $19.05 \times 9.53 \times 160$ & $10-15$ & 7.87 \\
WR-112( $\left(v_{2}\right)$ & $28.5 \times 12.6 \times 160$ & $7-10$ & 5.26 \\
\hline \hline
\end{tabular}

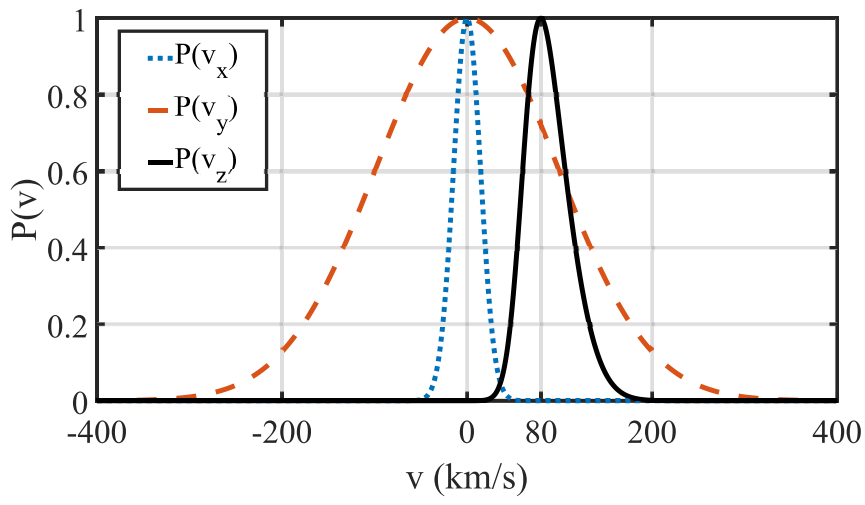

FIG. 2. Velocity distribution of excited-state atoms, including velocity selection in $v_{x}$ (dotted line) by the excitation laser and subsequent correlations in $v_{y}$ (dashed line) and $v_{x}$, as explained in the text. The solid line represents the $v_{z}$ distribution of Ps atoms.

The trajectories of excited atoms passing through the waveguide depend on the underlying velocity distributions of the emitted ground-state Ps atoms and velocity selection resulting from the bandwidth of the excitation laser (which is approximately $100 \mathrm{GHz}$ [18]). The physical structures within the vacuum chamber are all included in the simulated system geometry, and any Ps atoms that strike metal surfaces are deemed to be lost via annihilation. Ps atoms enter the waveguides through tungsten grids that have a geometrical open area of $92.5 \%$. Atoms lost in this way do not affect the simulations and have not been included.

The positron beam and laser systems are both pulsed, with widths of approximately 3 and $6 \mathrm{~ns}$, respectively [18]. However, the Ps density is low enough that no Ps - Ps interactions are possible [19], and simulations are therefore based on single atom trajectories.

The excited-state Ps velocity distributions used in the simulations are shown in Fig. 2. The ground-state Ps velocity distribution in the $x$ direction (see Fig. 1) is known from direct Doppler profile measurements [20] and, because of the random structure of the mesoporous silica film, the velocity profile in the $y$ direction is expected to be identical to this. However, for excited states the velocity distribution along the $x$ direction will be limited by the laser bandwidth, since the excitation laser propagates along this direction. The velocity distribution along the $z$ direction has been measured in separate experiments using Rydberg Ps atoms in a multiring electrostatic guide [21].

These profiles have been measured individually, but the full three-dimensional Ps velocity profiles are not known directly. The measured $v_{z}$ profiles are already those of excited-state atoms since they are measured using Rydberg atoms (the secondary excitation from $n=2$ to Rydberg states has a negligible effect on the velocity distributions).

Correlations between the $x, y$, and $z$ velocity components are estimated in the following way: the emission of Ps from silica is known to occur with energies on the order of $1 \mathrm{eV}$ [22]. However, in mesoporous films Ps then cools down via collisions with internal surfaces [20,23]. The cooling process becomes truncated when the Ps reaches the zero-point energy of the confining voids, and in the samples of the type used 


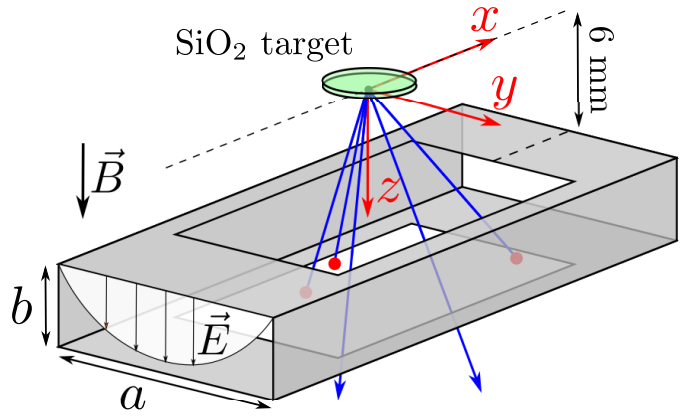

FIG. 3. Geometry of the numerical simulation. Positronium atoms are emitted from the $\mathrm{SiO}_{2}$ target at the origin of the coordinate system. Excited-state atoms fly along straight trajectories through the waveguide, where they interact with time-dependent electric and magnetic fields. Tungsten meshes in rectangular orifices of the waveguide (not shown in the figure) were present in the experiment but were not taken into account in the simulation.

in this work cooling below $\approx 40 \mathrm{meV}$ (in one dimension) is not observed, even if the samples are cooled to cryogenic temperatures [24]. Thus we assume that the average total Ps energy is approximately $120 \pm 30 \mathrm{meV}$, where the error is estimated from the maximum speeds observed. The distribution of pore size silica films can directly translate into a spread of Ps emission energies, arising from variations in the zero-point energy. Moreover, random interconnects and opening of the pore structures at the surface of the samples will also result in a variation in Ps energies.

The Ps velocities are generated by using a Monte Carlo selection of the Ps energy from the estimated range, and then a Monte Carlo sampling from the distributions shown in Fig. 2, limited by the total energy. This determines the ground-state Ps velocities, and for excited states an additional selection is then necessary to account for the finite laser bandwidth: atoms determined to have $v_{x}$ outside of the laser bandwidth cannot then be excited. Unless otherwise noted, in simulations we assume that atoms fly in straight trajectories with constant velocity $\vec{r}(t)=\vec{r}_{0}+\vec{v} t$, where $\vec{v}$ is the $3 \mathrm{D}$ velocity vector of the atom. Atoms fly from $z_{0}=0$ to $z_{1}=6 \mathrm{~mm}$ outside of the waveguide, then enter it, then fly out of it after the point $z_{2}=z_{1}+b$, where $b$ is a height of the waveguide (see Fig. 3).

\section{B. Numerical model overview}

The quantum state evolution of the atoms is computed using the master-equation approach described in detail in Sec. III. The initial state of the density matrix is set to a combination of the $2{ }^{1} S_{3}$ sublevels. During the flight on straight trajectories atoms are affected by time-dependent electric and magnetic fields $\vec{E}(\vec{r}, t)$ and $\vec{B}(\vec{r}, t)$. We compute the evolution of the density matrix during the flight with the time-dependent probability of annihilation used to extract a signal (see Sec. II C).

Since the waveguides used in the experiments were chosen such that the only allowed mode is the $\mathrm{TE}_{10}$ mode, we write the electric- and magnetic-field vectors inside the waveguide (using SI units) as

$$
\begin{aligned}
& \vec{E}_{ \pm}=E_{0}\left(\begin{array}{c}
0 \\
0 \\
\sin \left(\frac{\pi y}{a}\right) \cos \left( \pm k_{x} x+\omega t\right)
\end{array}\right), \\
& \vec{B}_{ \pm}=\frac{E_{0}}{a \omega}\left(\begin{array}{c}
-\pi \cos \left(\frac{\pi y}{a}\right) \sin \left( \pm k_{x} x+\omega t\right) \\
\pm k_{x} a \sin \left(\frac{\pi y}{a}\right) \cos \left( \pm k_{x} x+\omega t\right) \\
0
\end{array}\right),
\end{aligned}
$$

where $E_{0}$ is the peak electric field of the $\mathrm{rf}$ wave, $\omega=2 \pi \times$ $\left(v_{J}+\Delta v\right)$ is the frequency of the wave with detuning $\Delta v, a$ and $b$ are the width and height of the waveguide (see Fig. 3), and $k_{x}=\sqrt{\frac{\omega^{2}}{c^{2}}-\frac{\pi^{2}}{a^{2}}}$ is the wave vector of rf field. The \pm sign before $k_{x}$ chooses a direction of the traveling wave. A general rf field inside the waveguide is a linear superposition of a forward-traveling wave, described by fields $\vec{E}_{+}$and $\vec{B}_{+}$, and a backward-traveling wave, described by fields $\vec{E}_{-}$and $\vec{B}_{-}$. We assume no exposure to rf electric and magnetic fields outside the waveguide, keeping only a dc axial magnetic field of $32 \mathrm{G}$ (if necessary).

The numerical simulation allows us to study various systematic effects by including necessary elements in the model and removing the elements which may hide them. Within this paradigm, to investigate linewidth and quantum interference shifts, we consider a model of an ideal forward-traveling wave with constant amplitude. To illustrate a reflection-related distortion of the linewidth, we consider a combination of the forward-traveling wave and a small reflection from a fixed distance. The most realistic simulation is based on numerical computation of the rf field distribution (see Sec. IV) used to extract parameters of forward- and backward-traveling waves.

\section{Signal extraction}

In the experiments [6] Ps decay rates were measured using the technique of single-shot positron annihilation lifetime spectroscopy (SSPALS) [25]. This method allows Ps lifetime spectra to be generated when many gamma-ray photons are detected nearly simultaneously. The observed signals are equivalent to a measurement of the annihilation radiation intensity as a function of time. These data are extracted as integrals of the current through photomultiplier (PMT) detectors, which we define as

$$
V_{A T}=\int_{A}^{T} I(t) d t,
$$

where $A$ is the moment of time related to the beginning of the measurement cycle and $I$ is a current output of the PMT. The experimental procedure [25] involves extracting a parameter $f_{d}$, that is proportional to the fraction of long-lived atoms, using

$$
f_{d}=\frac{V_{A C}-V_{A B}}{V_{A C}},
$$

where the times $B$ and $C$ were set to $700 \mathrm{~ns}$ and $1400 \mathrm{~ns}$ after the beginning of the measurement cycle, respectively. In the experiment, the fraction of long-lived atoms was measured with $\left(f_{\text {on }}\right)$ and without $\left(f_{\text {off }}\right)$ microwave radiation present. 


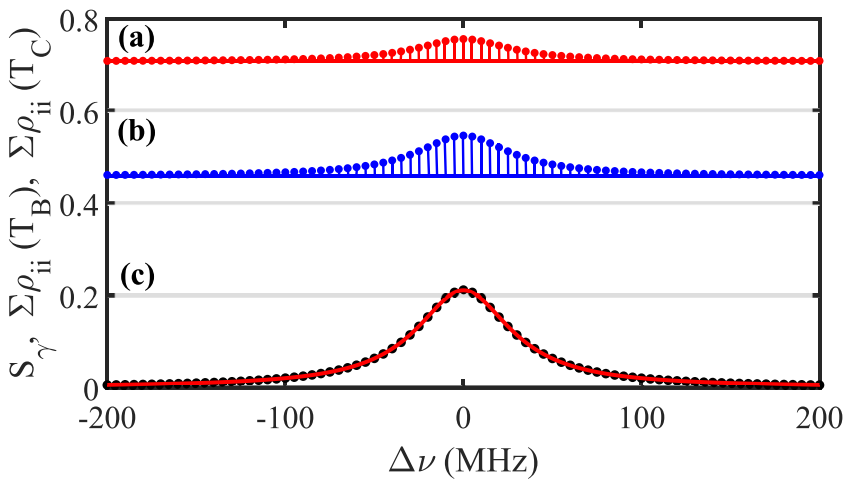

FIG. 4. Example of procedure of signal $S_{\gamma}$ calculation. (a) The fraction of "long-lived" atoms $\sum \rho_{i i}\left(T_{C}\right)$, which annihilate from $t=0$ to $T_{C}=1400 \mathrm{~ns}$, the analog of experimentally measured value $V_{A C}$. (b) The fraction of the "short-lived" atoms $\sum \rho_{i i}\left(T_{B}\right)$, which annihilate from $t=0$ to $T_{B}=700 \mathrm{~ns}$, the analog of experimentally measured value $V_{A B}$. Points on both blue and red result for the "rf on" regime with frequency and the solid line for the "rf off" regime. (c) Black points: signal $S_{\gamma}$ calculated using Eqs. (3), (4), and (5). Red solid line: Lorentz fit of the calculated line shape.

The microwave radiation resonantly mixes long-lived $2^{3} S_{1}$ with $2^{3} P_{J}$ states, causing faster decay of atoms to the $1^{3} S_{1}$ state, with fast annihilation from the ground state thereafter. A dimensionless quantity used as the signal is defined as

$$
S_{\gamma}=\frac{f_{\text {off }}-f_{\text {on }}}{f_{\text {off }}} .
$$

To simulate the signal, we introduce in the density matrix a set of fictitious "annihilation states" to count annihilation events. The initial population of all those states is set to zero, so the integrals of current through detectors in experiment are related to the sum

$$
V_{A T}=\sum_{i \in \mathbf{A}} W_{i} \rho_{i i}(T),
$$

where $\rho_{i i}$ refers to density-matrix elements (see Sec. III B), and the sum can be taken with different weights $W_{i}$, so as to allow investigations of the sensitivity of line positions with different detector orientations.

Figure 4 shows an example of a calculation of the signal using simulated frequency-dependent $V_{A C}$ and $V_{A B}$ values. The resulting signal fits a nonshifted Lorentzian function (red bottom line), confirming that, in the ideal case, the simulations reproduce the expected transition frequencies.

\section{Line-shape fitting}

As in the experimental case, two types of fit function were used to characterize the simulated data-the symmetric Lorentzian function

$$
S_{L}=b_{L}+\frac{a_{L}}{1+4\left(\frac{v-v_{L}}{w_{L}}\right)^{2}}
$$

and the Fano function [26]

$$
S_{F}=b_{F}+a_{F} \frac{1+2 \eta\left(\frac{v-v_{F}}{w_{F}}\right)}{1+4\left(\frac{v-v_{F}}{w_{F}}\right)^{2}},
$$

which is obtained from the form given in the experimental article [6] by the replacements $\eta=\frac{2 q}{q^{2}-1}, a_{F}=\frac{A\left(q^{2}-1\right)}{\left(q^{2}+1\right)}$, and $b_{F}=\frac{A}{q^{2}+1}$. The Fano fit in that form was used to describe quantum interference effects in hydrogen spectroscopy experiments [13] as proposed in Ref. [27]. The $b_{L, F}$ and $a_{L, F}$ parameters are responsible for the signal background and amplitude, respectively, $v_{L, F}$ is the center of the line, and $w_{L, F}$ parameters characterize the linewidth. In the limit $\eta \rightarrow 0$, the Fano fit function approaches the Lorentzian one. The Fano function is used to characterize quantum interference effects on the line shape. This is in contrast to the experimental case [6], in which the Fano function was used only as a way to characterize asymmetry, with no underlying physical basis.

As a qualitative parameter that characterizes an asymmetry, we use a discrepancy between line center frequency obtained from Lorentz and Fano fits. Another parameter that may be used to characterize the asymmetry is the parameter $\eta$ in the Fano line shape (7). A rough evaluation of the connection between those two methods gives a relation $v_{L}-v_{F} \approx \eta w_{L} / 2$. In order to understand the line-shape asymmetry we pay special attention to the parameter $\eta$ of the Fano fit of simulated data as well as the line center discrepancy $v_{L}-v_{F}$.

\section{E. Linewidth of the transition}

In the framework of this paper, line-shape distortions are characterized by only two parameters - shifts and broadening; more elaborate line-shape descriptions cannot be validated owing to the limited signal-to-noise ratio of the measured data. We therefore discuss only centroid shifts and linewidths extracted from Lorentz and Fano fits to the simulated data. Line shifts are the most important properties of these fits with respect to understanding the measured fine-structure intervals, with concomitant corrections or uncertainties for the experimental error budget. However, linewidths do provide some insight into the experiments and can be used to cross-check saturation data and Ps velocity distributions.

The natural linewidth of $2 S-2 P$ transitions in Ps is $\approx$ $50 \mathrm{MHz}$, primarily determined by the $3.19 \mathrm{~ns}$ radiative lifetime of the $2 P$ levels. The linewidth observed in the experiments [6] is about 62-66 MHz. In this section, we consider the mechanisms of additional broadening and the experimental parameters affecting it.

\section{Doppler broadening}

Doppler broadening may arise from variations of the Ps velocity component $v_{x}$ in the direction of the waveguide. The frequency of the $\mathrm{rf}$ field observed by a moving atom with $x$ component of the velocity $v_{x}$ can be expressed as $-k_{x} v_{x}$, which causes a shift of the line center frequency by $+k_{x} v_{x}$. To simulate line distortions caused by this effect we calculate line shapes for a regular set of $v_{x}$ from $-70 \mathrm{~km} / \mathrm{s}$ to $+70 \mathrm{~km} / \mathrm{s}$. Using weighted averaging, we can obtain an approximate line shape for an arbitrary velocity distribution in the computed interval. The $v_{x}$ distribution (see Fig. 2) can be approximated by a Gaussian profile $\exp \left[-v_{x}^{2} / v_{w}^{2}\right]$ with width parameter $v_{w} \approx 20 \mathrm{~km} / \mathrm{s}$.

To study the effect of Doppler broadening, we compute how the FWHM $w_{L}$ of the line profile obtained from Lorentz 


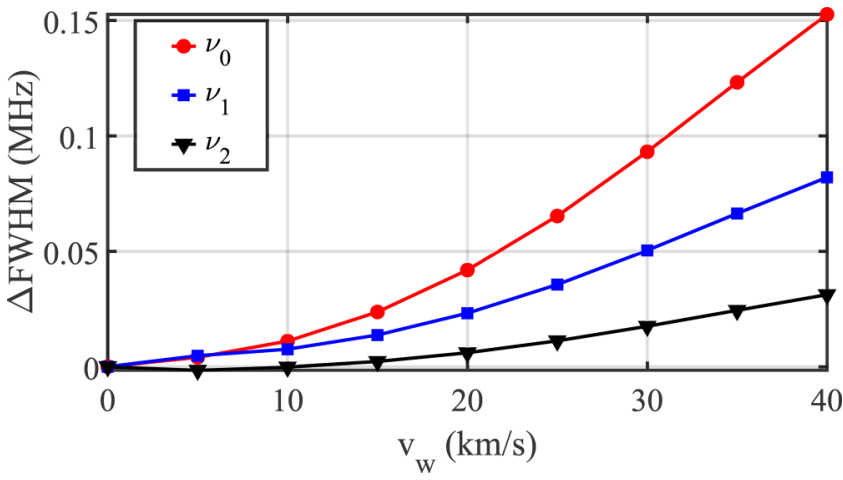

FIG. 5. Doppler broadening, illustrated by the difference between the FWHM of Lorentz fits of the line without $v_{x}$ velocity averaging and with $v_{x}$ velocity averaging, computed for $v_{0}$ (circles), $v_{1}$ (squares), and $v_{2}$ (triangles) transitions vs the velocity distribution spread $v_{w}$.

fits depends on $v_{w}$ in a broad range around the most probable value of $20 \mathrm{~km} / \mathrm{s}$. The results presented in Fig. 5 indicate that the Doppler broadening is negligible in comparison with other broadening mechanisms.

\section{Time-of-flight broadening}

To study time-of-flight broadening, we compute line shapes for $v_{x}=0$ (to remove Doppler broadening) and $20 \mathrm{~dB}$ lower rf power than was used in the experiment (to suppress saturation broadening). The linewidth of Lorentz fits for the computed line shapes for various $v_{z}$ values is shown in Fig. 6. For high velocities, broadening depends on the time-of-flight $b / v_{z}$, so the linewidth also varies with the height of the waveguides $b$. For the most probable velocity of about $80 \mathrm{~km} / \mathrm{s}$, the time-of-flight broadening is about $3 \mathrm{MHz}$ (for the $v_{0}$ transition).

Slow velocity groups also may experience broadening because all atoms have to pass the $6 \mathrm{~mm}$ gap between target and waveguide within the $700 \mathrm{~ns}$ time window. The slow atoms spend too much time in the gap, which shortens inter-

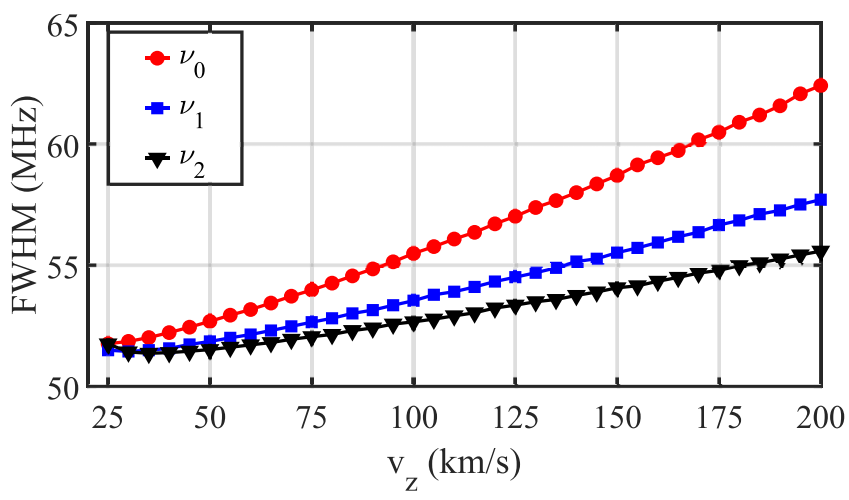

FIG. 6. Time-of-flight broadening, illustrated by the FWHM of Lorentz fit of the lines computed for $v_{0}$ (circles), $v_{1}$ (squares), and $v_{2}$ (triangles) transitions vs the velocity $v_{z}$ of the atom. The broadening of the lines for higher velocities is caused by time-of-flight broadening concerned with the dimensions of the waveguide.

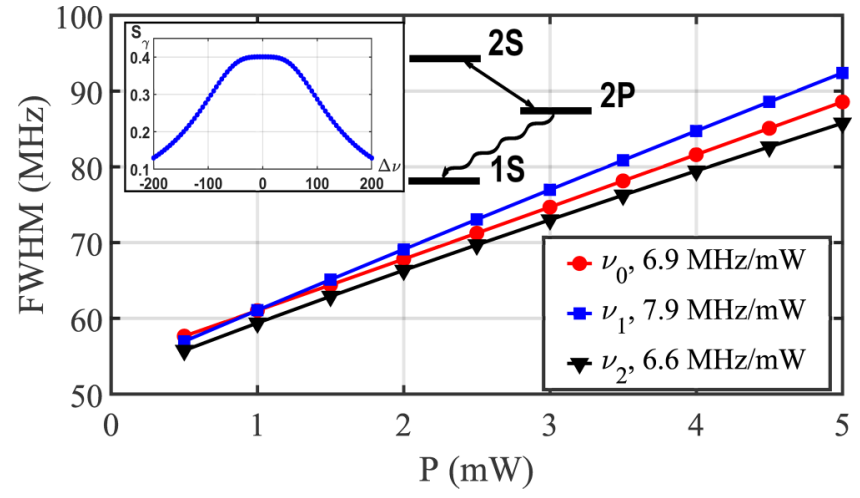

FIG. 7. Saturation broadening, illustrated by the FWHM of Lorentz fit of the lines computed for $v_{0}$ (circles), $v_{1}$ (squares), and $v_{2}$ (triangles) transitions vs the power $P$ in $\mathrm{mW}$ of the wave inside the waveguide. The line shapes were computed for the velocity distributions presented in Fig. 2. The broadening of the lines for higher powers is caused by the saturation of the transition. The noncyclic level structure of positronium (top) implies depletion of the $2 S / 2 P$ levels population; therefore, vast rf power causes flat-top line distortion. Inset plot: the line shape of $v_{1}$ transition computed for microwave power of $P=50 \mathrm{~mW}$ manifests flat-top distortion.

action time and produces an effect similar to the time-of-flight broadening.

\section{Saturation broadening}

The large amplitude of the electromagnetic wave causes line distortion, which also appears as a broadening. The most well-known example is a simple power broadening in twolevel systems (e.g., Ref. [28]). This mechanism causes an increase of the linewidth of the transition while its shape stays Lorentzian. Also, this broadening type depends only on the wave's amplitude, and it does not depend on the interaction time. In contrast, driving the experiments $2 S-2 P$ transition causes depletion of $2 S / 2 P$ states population via decay to $1 S$. The depletion related to power and interaction time leads to non-Lorentzian line distortion. For high rf amplitude this distortion appears as a "flat-top" effect (see Fig. 7). Unlike the power broadening in two-level systems, this distortion depends on the time that atoms stay in the field. A mathematically precise description of this distortion is complex, but we may characterize it using a Lorentz fit of the computed line shapes.

The peak electric field $E_{0}$ of the traveling wave (1) is connected with the power $P$ with the following relation:

$$
E_{0}^{2}=\frac{4 P}{a b c \epsilon_{0} \sqrt{1-\frac{\pi^{2} c^{2}}{a^{2} \omega^{2}}} .}
$$

To study the saturation broadening, we compute line shapes, averaging over the $v_{z}$ and $v_{x}$ velocities for powers of the microwave field ranging from 0.5 to $5 \mathrm{~mW}$. The results are shown in Fig. 7 and can be linearly fitted with good accuracy. The 3 to $5 \mathrm{MHz}$ difference from the natural linewidth at zero power is caused by time-of-flight broadening. The fit slopes depend on $v_{z}$ velocity distribution and are given by $6.9 \mathrm{MHz} / \mathrm{mW}, 7.9 \mathrm{MHz} / \mathrm{mW}$, and $6.6 \mathrm{MHz} / \mathrm{mW}$ for $v_{0}, v_{1}$, 
and $v_{2}$ transitions, respectively. In the rf power of more than $1 \mathrm{~mW}$, this saturation is the leading broadening mechanism. Our simulation shows that other mechanisms manifest themselves as nonzero broadening at $P \rightarrow 0$, but they are negligible compared to the saturation effect. Therefore, the observed linewidth can be used to estimate the actual power of the rf wave coupled into the waveguide.

\section{QUANTUM DYNAMICS AND INTERFERENCE}

\section{A. Master equation}

The quantum dynamics of positronium atoms are described using numerical integration of a master equation in the Lindblad form [15] (see also Refs. [29,30]). The master equation describes the evolution of the density matrix of the atom in the form of an ordinary differential equation:

$$
\frac{d \rho}{d t}=-\frac{i}{\hbar}[\hat{H}, \rho]+\mathcal{L} \rho,
$$

where $\rho$ is a density matrix of the system, $\hat{H}$ is the Hamiltonian of the system, and $\mathcal{L} \rho$ is a spontaneous relaxation superoperator. We describe this superoperator as

$$
\mathcal{L} \rho=-\sum_{\alpha, \beta \in D} \frac{\gamma_{\alpha \beta}}{2}\left(\rho S_{\alpha}^{+} S_{\beta}^{-}+S_{\alpha}^{+} S_{\beta}^{-} \rho-2 S_{\beta}^{-} \rho S_{\alpha}^{+}\right),
$$

where $D$ represents the set of all possible spontaneous decay channels in the considered atomic system, $\gamma_{\alpha \beta}$ is a crossdamping constant for two different decay channels $\alpha$ and $\beta$, $S_{\alpha, \beta}^{+}$are operators of excitation of decay channel, and $S_{\alpha, \beta}^{-}$are deexcitation operators. In our model we describe each decay channel $\alpha$ by its upper state $\left|u_{\alpha}\right\rangle$, lower state $\left|l_{\alpha}\right\rangle$, and an offdiagonal electric dipole moment $\vec{d}_{\alpha}=\left\langle l_{\alpha}|\hat{\vec{d}}| u_{\alpha}\right\rangle$, associated with this decay. In this description we write excitation and deexcitation operators as $S_{\alpha}^{+}=\left|u_{\alpha}\right\rangle\left\langle l_{\alpha}\right|$ and $S_{\alpha}^{-}=\left|l_{\alpha}\right\rangle\left\langle u_{\alpha}\right|$. The cross-damping constant (in SI units) is

$$
\gamma_{\alpha \beta}=\frac{\omega_{\alpha}^{3 / 2} \omega_{\beta}^{3 / 2}\left(\vec{d}_{\alpha}, \vec{d}_{\beta}\right)}{3 \pi \epsilon_{0} \hbar c^{3}},
$$

where $\omega_{\alpha}$ is a frequency of transition, $\epsilon_{0}$ is a vacuum permittivity, and $c$ is the speed of light. One can see that, if $\alpha=\beta$, Eq. (11) represents a standard electric dipole allowed decay constant. If $\alpha \neq \beta$, Eq. (11) describes a constant responsible for the quantum interference of two decay channels.

\section{B. Basis states and Hamiltonian}

We model a positronium atom in the basis of its quantum states which are the eigenfunctions of its unperturbed Hamiltonian (see Fig. 8). A convenient set of quantum numbers for us is $|n, S, L, J, M\rangle$, where $n$ is the principal quantum number, $S$ is the sum of electron and positron spins, $L$ is the orbital angular momentum quantum number, $J$ is the total angular momentum quantum number (i.e., the sum of $S$ and $L$ ), and $M$ is a projection of the total angular momentum on a quantization axis. The direction of the quantization axis can be defined arbitrarily; here we always use the $z$ axis of the experimental geometry. We restrict our basis to all quantum states with $n=1,2$, neglecting a population of $n>2$ levels. This corresponds to the experiment in which the atoms formed

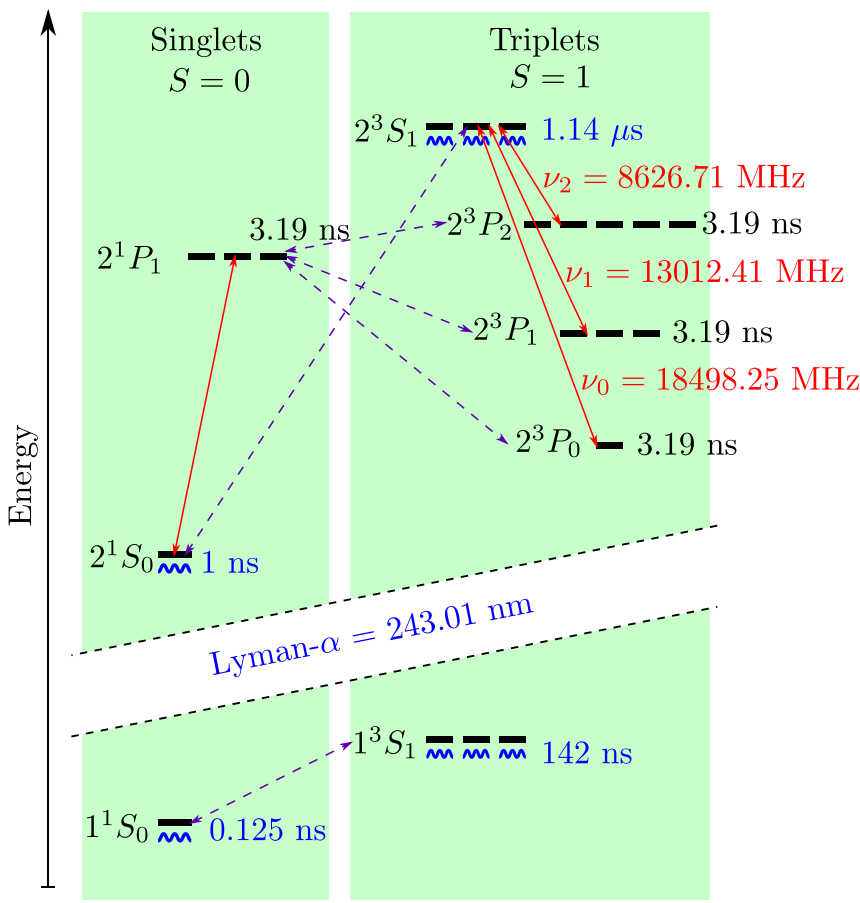

FIG. 8. Diagram of quantum states of positronium used in the simulation model. States marked with blue wavy lines are fictitious states for counting annihilation events. Red (solid) arrows represent electric dipole allowed transitions; violet (dashed) arrows represent magnetic dipole allowed transitions. Spontaneous decays $2 P \rightarrow 1 S$ are included in the simulation but not shown here. Radiative or annihilation lifetimes for all levels are also indicated, according to the primary decay mode.

in the ground state $n=1$ are excited to state $n=2$ by a narrow-band laser. The laser excitation allows us to avoid excitation of $n>2$ levels, which may produce additional quantum interference shifts similar to that described in work [31]. The diagram of those states is shown in Fig. 8; it includes 20 real quantum states of the positronium atom, i.e., 16 states in the $n=2$ manifold and four states in the $n=1$ manifold.

To model the signal detected in the experiments we introduce additional fictitious states ("annihilation states") to count annihilation events. We neglect the possibility of annihilation of $2 P$ states [32,33], so it is sufficient to include eight such states, corresponding to real states with $L=0$. We thus model the annihilation process as a decay from a real state $|n, S, L, J, M\rangle$ to an annihilation state $|n, S, L, J, M\rangle^{A}$, with a decay constant corresponding to that of the real state. We assume that all eight annihilation channels do not interfere, which happens automatically in the model because the final states are distinguishable. The fictitious states are included in the basis, extending our density-matrix size to $28 \times 28$. However, they are not included in the Hamiltonian, and their role in the numerical model is limited by a final state of cascade decay processes.

The Hamiltonian of the positronium atom is written as

$$
\hat{H}=\hat{H}_{0}-\hat{\vec{d}} \vec{E}(\vec{r}, t)-\hat{\vec{\mu}} \vec{B}(\vec{r}, t)
$$


where $\hat{H}_{0}$ is the unperturbed Ps Hamiltonian, $\vec{E}$ is the classical electric field, $\vec{r}$ is a coordinate of the atom at time $t, \hat{\vec{d}}$ is an electric dipole moment operator, $\vec{B}$ is a classical magnetic field, and $\hat{\vec{\mu}}$ is a magnetic dipole moment operator. The Hamiltonian $\hat{H}_{0}$ is written as a diagonal matrix; the energies of the states are taken from theory (see [4] and references therein). Both electric and magnetic fields are written explicitly as functions of time and coordinate, which allows us to include dc fields and rf fields in a convenient form.

To avoid fast oscillation of optical frequencies, which would make numerical integration of the master equation impossible, we use the rotating wave approximation (RWA) (e.g., Ref. [28]). We make the following substitution of variables for density-matrix elements $\rho$ in Eq. (9):

$$
\rho_{i j}=\tilde{\rho}_{i j} \exp \left\{-\left(\frac{i}{\hbar}\right)\left(E_{i}-E_{j}\right) t\right\},
$$

where $E_{i}=E_{1 S}$ if $i$ belongs to the $n=1$ manifold and $E_{i}=$ $E_{2 S}$ if $i$ belongs to the $n=2$ manifold. The energies $E_{1 S}$ and $E_{2 S}$ are mean energies of $n=1$ and $n=2$ manifolds. It is obvious that we make such a replacement only for fastoscillating $\rho_{i j}$, where $i$ and $j$ are related to different manifolds; this operation replaces them with slow-oscillating variables $\tilde{\rho}_{i j}$. After the substitution (13) we find that Eq. (9) is in a form where all fast oscillations are written explicitly as exponential factors. We implement the RWA by neglecting all the terms which oscillate with a frequency above $1 \mathrm{THz}$. The resulting system of equations is suitable for numerical integration. This method works if we use as $E_{n s}$ any energy, close to the energies of manifold, because the wrong selection of $E_{n S}$ will only affect the performance of the numerical code and not the final output of that code.

Since our basis consists of 28 different states, system (9) contains $28^{2}=784$ coupled differential equations. To derive them, we use a dedicated $\mathrm{C}++$ program that uses the computing algebra system GINAC [34]. The system of equations was cross-checked via a similar derivation in Wolfram Mathematica script. We also applied an analytic simplification of the system, which helps us to remove a large fraction of density-matrix variables, leaving only 408 coupled differential equations. The derived system of equations was transformed to $\mathrm{C}++$ compatible code incorporated into a fast numerical solver, using the Runge-Kutta eighth order method with Dormand-Prince step prediction algorithm [35]. The numerical integration code solves the differential equations for zero rf field (to obtain $f_{\text {off }}$ ) and for each possible value of the rf frequency, giving as output a table of all values of the annihilation state population. These data were then used to obtain a signal using Eqs. (3), (4), and (5).

\section{Quantum interference}

Quantum interference is an effect causing distortion of line shapes due to the presence of other nearby nonresonant levels in the atomic system. Shifts associated with this effect have been widely discussed (e.g., Refs. [11,13,36-38]). Such shifts may arise from the interference of quantum evolution paths through resonant levels with paths through nonresonant levels. The phase relations which affect the interference are connected with the polarization of exciting and emitted light.
As a result, the QI shift often manifests itself as a dependence of the line center from the relative polarization of the exciting electromagnetic field and detected spontaneous relaxation photons (e.g., Ref. [12]).

In contrast to many previous works, the signal in the positronium experiment does not come from fluorescence decay photons, but rather from annihilation photons. However, these annihilation events are preceded by Lyman $\alpha$ decays, and the polarization of the emitted $243 \mathrm{~nm}$ photons is entangled with the spin projection $M$ of the final $1 S$ state. If the $\gamma$-quanta detectors have various sensitivity to annihilation from different Zeeman components of $1{ }^{3} S_{1}$ level, there will be a QI shift.

In this section, we first consider a simple model based on the Kramers-Heisenberg formula to illustrate the nature of the QI shift. The result depends on the geometrical properties of the detectors, and a simplified model of this mechanism is described below. We then, in Sec. IIIC2, use the master-equation approach to obtain a more realistic estimation of the QI shift in the positronium experiment.

\section{Simple model of QI shift}

To illustrate the QI shift [see Fig. 9(a)] let us consider a simplified case where an atom in an initial state $|i\rangle=$ $\left|2^{3} S_{1}(M=0)\right\rangle$ interacts with a $z$-polarized rf electric field. The rf electric field drives transitions to excited states $\left|e_{1,2}\right\rangle=$ $\left|2^{3} P_{J}(M=0)\right\rangle$, with $J=0,2$ (the transition to $J=1, M=$ 0 is forbidden by electric dipole selection rules). Excited states decay to a manifold of final states $\left|f_{1,2,3}\right\rangle=\mid 1^{3} S_{1}(M=$ $-1,0,+1)\rangle$. The probability of process $i \rightarrow f_{\alpha}$ should be derived as a result of interference of two paths: $i \rightarrow e_{1} \rightarrow f_{\alpha}$ and $i \rightarrow e_{2} \rightarrow f_{\alpha}$.

Note that the frequency difference between $2{ }^{3} P_{0}$ and $2{ }^{3} P_{2}$ states is about $10 \mathrm{GHz}$, while the natural linewidth is about $50 \mathrm{MHz}$. Thus the lines of transitions $i \rightarrow e_{1}$ and $i \rightarrow e_{2}$ are separated by $\approx 200$ linewidths, so the presence of a distant level may seem to be negligible. However, significant interference effects are possible even with such a large separation of levels (see, e.g., Ref. [11]). Following the approach used in Refs. [12,36], we can write a rate of spontaneous photon emission via the Kramers-Heisenberg formula as

$$
R_{q, p}(i \rightarrow f)=\frac{\pi E_{0}^{2} \omega_{s}^{3}}{h^{3} c^{3} \epsilon_{0}}\left|\sum_{e} \frac{d_{p}(i \rightarrow e) d_{q}(e \rightarrow f)}{\omega-\omega_{e i}+i \Gamma / 2}\right|^{2}
$$

where $q$ denotes polarization of emitted photons, $p$ is a polarization of rf electric field ( 0 for $\pi$-polarized field and \pm 1 for $\sigma^{ \pm}$-polarized field), $E_{0}$ and $\omega$ are electric-field amplitude and frequency, $\omega_{s} \approx 2 \pi \times 1230 \mathrm{THz}$ is emitted photons' frequency, $d_{p}(i \rightarrow e)$ and $d_{q}(e \rightarrow f)$ are emission dipole moments, $\omega_{e i}=\omega_{i}-\omega_{e}$ is energy difference between $i$ and $e$ states, and $\Gamma$ is an inverse lifetime of state $e$. In our simple example we have only two excited states $e_{1}$ and $e_{2}$. To continue, we designate $\mathrm{rf}$ detuning $\delta=\omega-\omega_{e_{1} i}$ and level separation $\Delta=\omega_{e_{2} i}-\omega_{e_{1} i}$. Then, since the polarization of the exciting electric field is directed along the $z$ axis, we set $p=0$. The dipole moments for $i \rightarrow e$ transitions can be easily calculated 
(a)
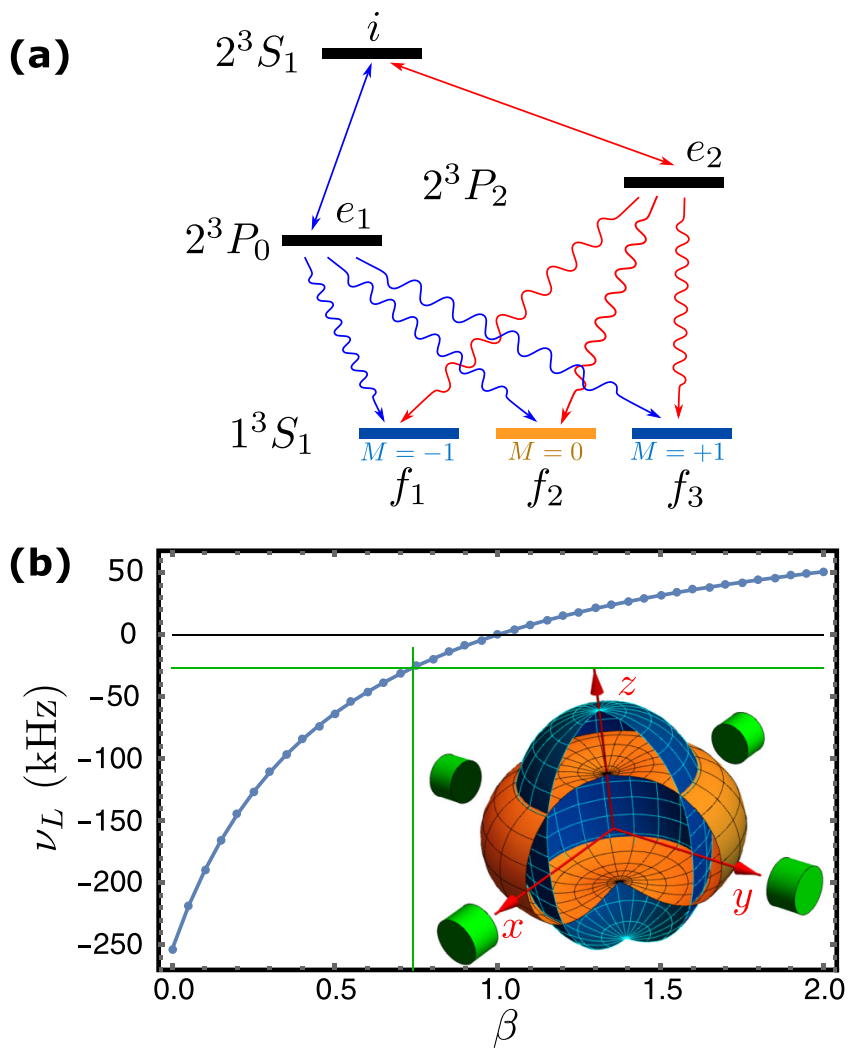

FIG. 9. (a) Simple scheme to illustrate QI shift in the positronium experiment. We compute rate $R_{k}$ of transitions $i \rightarrow e_{1} \rightarrow f_{1,2,3}$ (blue arrows) as a function of driving field frequency. The presence of off-resonant paths $i \rightarrow e_{2} \rightarrow f_{1,2,3}$ (red arrows) leads to interference which shifts the lines. (b) Lorentz fit center $v_{L}$ of the function $R_{2}+\beta\left(R_{1}+R_{3}\right)$. For $\beta=1$ detection probabilities of Zeeman components $M= \pm 1$ and $M=0$ are equal and the QI shift vanishes. For the realistic case $\beta=0.74$ the QI shift computed with this simple model is about $-26.5 \mathrm{kHz}$. Inserted figure: spatial distribution of the annihilation photons with respect to quantization axis $z$ : blue/bright mesh for $M= \pm 1$ component; orange/dark mesh for $M=0$ component. Detectors (green) placed in the $x y$ plane are more sensitive to the $M=0$ component with weight ratio approximately $0.74: 1: 0.74$.

(in atomic units of $e a_{0}$ ):

$$
\begin{aligned}
& d_{0}\left(i \rightarrow e_{1}\right)=+2 \sqrt{3}, \\
& d_{0}\left(i \rightarrow e_{2}\right)=-2 \sqrt{6} .
\end{aligned}
$$

The dipole moments for $e \rightarrow f$ can also be simply computed:

$$
\begin{aligned}
d_{+1}\left(e_{1} \rightarrow f_{1}\right)=d_{-1}\left(e_{1} \rightarrow f_{3}\right) & =+\frac{256}{243} \sqrt{\frac{2}{3}}, \\
d_{+1}\left(e_{2} \rightarrow f_{1}\right)=d_{-1}\left(e_{2} \rightarrow f_{3}\right) & =+\frac{256}{243} \sqrt{\frac{1}{3}}, \\
d_{0}\left(e_{1} \rightarrow f_{2}\right) & =-\frac{256}{243} \sqrt{\frac{2}{3}}, \\
d_{0}\left(e_{2} \rightarrow f_{2}\right) & =+\frac{512}{243} \sqrt{\frac{1}{3}} .
\end{aligned}
$$

Substituting these numbers into Eq. (14), we can get approximate line shapes of transitions to states $f_{1}, f_{2}$, and $f_{3}$ neglecting constant factor:

$$
\begin{array}{r}
R_{1}=R_{3} \sim S_{1}+S_{2}-2 S_{12}, \\
R_{2} \sim S_{1}+4 S_{2}+4 S_{12},
\end{array}
$$

where $R_{1}=R_{0,+1}\left(i \rightarrow f_{1}\right), R_{2}=R_{0,0}\left(i \rightarrow f_{2}\right)$, and $R_{3}=$ $R_{0,-1}\left(i \rightarrow f_{3}\right)$ are transition rates to $f_{1,2,3}$ states. The symbols $S_{1}, S_{2}$, and $S_{12}$ designate the Lorentz line shape for $i \rightarrow e_{1}$ transition, $i \rightarrow e_{2}$, and their interference:

$$
\begin{gathered}
S_{1}=\frac{1}{\Gamma^{2}+4 \delta^{2}}, \\
S_{2}=\frac{1}{\Gamma^{2}+4(\delta+\Delta)^{2}}, \\
S_{12}=\frac{2\left[\Gamma^{2}+4 \delta(\delta+\Delta)\right]}{\left(\Gamma^{2}+4 \delta^{2}\right)\left[\Gamma^{2}+4(\delta+\Delta)^{2}\right]} .
\end{gathered}
$$

The term (20) leads to distortion of the line shape, giving a QI shift. One can see that if $\Delta \gg \Gamma$ and $\Delta \gg \delta$ the interference term can be approximated by the dispersive part of the Fano profile:

$$
S_{12} \approx \frac{2}{\Delta} \frac{\delta}{\Gamma^{2}+4 \delta^{2}} .
$$

Due to this, using Fano profiles instead of Lorentz profiles includes the interference term and helps to avoid the QI shift. Another way to avoid the interference term is a proper weighting of the signal components. From Eq. (17) one can see that if we detect annihilation from states $f_{i}$ with equal probabilities the line shape is

$$
R_{1}+R_{2}+R_{3} \sim 3 S_{1}+6 S_{2} .
$$

This line has no QI shift since the $S_{12}$ term vanishes. Similarly, we can compute the shift for atoms that start from $2^{3} S_{1}(M= \pm 1)$ states and show the same property of the QI shift. In the real experiment, the detection probability of various Zeeman components is slightly different due to geometrical effects. Figure 9(b) shows how the Lorentz fit center of the line described by function $\beta\left(R_{1}+R_{3}\right)+R_{2}$ depends on weighting parameter $\beta$. To estimate the realistic value of $\beta$, we need to introduce the geometrical model of the detector.

In the experiments annihilation events are detected via the annihilation $\gamma$ quanta, meaning that we must include in the model some probability of event detection. In this paper we neglect the sensitivity of detectors to the energy and polarization of the annihilation photons, and consider only geometric effects. The directional emission profiles of $\gamma$ quanta from three-photon annihilation are [39]

$$
\begin{gathered}
P_{M=0} \sim\left(\pi^{2}-9\right) \sin ^{2}(\theta)+\frac{1}{6}\left[3 \cos ^{2}(\theta)-1\right], \\
P_{M=1} \sim\left(\pi^{2}-9\right)\left[\cos ^{2}(\theta)+1\right]-\frac{1}{6}\left[3 \cos ^{2}(\theta)-1\right] .
\end{gathered}
$$

The detectors in the real experiment are positioned around the setup orthogonal to the quantization axis [see Fig. 9(b)]. This breaks the symmetry, so the probabilities of annihilation detection of $M=-1, M=0$, and $M=+1$ states are different. For example, for pointlike detectors in the $x y$ plane they are related as $\left[6 \pi^{2}-53\right]:\left[12 \pi^{2}-110\right]:\left[6 \pi^{2}-53\right] \approx$ 
TABLE II. Difference between the line fit centers obtained by Lorentz $\left(v_{L}\right)$ and Fano $\left(v_{F}\right)$ fits from the unperturbed transition frequency used in the numerical simulations. The weights are the relative probabilities $W_{i}$ of positronium annihilation detection with spin projection $M=-1, M=0$, and $M=+1$, respectively [according to Eq. (5)]. The lines designated HP were calculated for the high-power regime, which is close to the best known estimation of rf power in experiment; the lines designated LP were calculated for the low-power regime, with an artificial decrease of power in the simulation by a factor of 100 to avoid saturation-related distortion of the line shape. The uncertainty of numerical calculation is less than $1 \mathrm{kHz}$.

\begin{tabular}{|c|c|c|c|c|c|}
\hline \multirow[b]{2}{*}{ Weights } & \multirow[b]{2}{*}{ Line } & \multicolumn{2}{|c|}{$v_{L}(\mathrm{kHz})$} & \multicolumn{2}{|c|}{$v_{F}(\mathrm{kHz})$} \\
\hline & & HP & LP & HP & LP \\
\hline \multirow{3}{*}{ 1:1:1 } & $v_{0}$ & -2.3 & 0.07 & -1.3 & 0.40 \\
\hline & $v_{1}$ & -1.8 & -0.07 & -1.2 & 0.06 \\
\hline & $v_{2}$ & 4.3 & -0.06 & 3.7 & -0.38 \\
\hline \multirow{3}{*}{ 1:0:0 } & $v_{0}$ & -249 & -138 & 29 & 0.76 \\
\hline & $v_{1}$ & 229 & 231 & -85 & -2.5 \\
\hline & $v_{2}$ & -150 & -133 & 67 & 0.82 \\
\hline \multirow{3}{*}{ 0:1:0 } & $v_{0}$ & 174 & 198 & -61 & -2.1 \\
\hline & $v_{1}$ & -618 & -304 & 170 & 2.0 \\
\hline & $v_{2}$ & 193 & 113 & -127 & -1.9 \\
\hline \multirow{3}{*}{ 0.74:1:0.74 } & $v_{0}$ & 34 & 25 & -8 & -0.21 \\
\hline & $v_{1}$ & -59 & -41 & 17 & 0.40 \\
\hline & $v_{2}$ & 29 & 18 & -10 & -0.57 \\
\hline
\end{tabular}

0.74:1:0.74. Precise measurement of the detection probabilities is difficult, so we restrict our consideration to extreme cases of maximal sensitivity to one of those components and the case of pointlike detectors.

\section{QI shift from the master equation}

Quantum interference is naturally included in the master equation, so we can use it to characterize this effect. We simulate experimental lines using a single running wave $\left(\vec{E}_{+}, \vec{B}_{+}\right)$without reflections, a constant electric-field amplitude $E_{0}(\omega)=$ const, and the velocity distributions shown in Fig. 2. We fit simulated line shapes with both Lorentz and Fano profiles, and compute the difference of the obtained fit centers from the transition frequency used in the simulation according to Eqs. (6) and (7). We consider this difference $v_{L}$ or $v_{F}$ as an estimate of the uncertainty caused by the line-shape model (or, more properly, the physical mechanisms described by those models). Similarly, the difference between the Lorentz and Fano fit centers can be considered as a metric of the asymmetry of the underlying line shape; that is, for symmetric line shapes they will be identical.

We summarize the results of this procedure in Table II. We observed that the results depend on at least two essential parameters: (1) the weights of the different Zeeman components and (2) the rf power. In the former case, the effects due to weighting the Zeeman components [i.e., $W_{i}$ in Eq. (5)] are a direct analog of shifts observed in experiments $[13,36]$ with limited solid angle detectors. Setting $W_{i}=1$ for all components effectively cancels out the QI shift, as shown above in the simple model. In the latter case, the rf power dependence arises from saturation of the transition, because the process
$2{ }^{3} S_{1} \rightarrow 2 P_{J} \rightarrow 1{ }^{3} S_{1}$ is not cyclic. Saturation causes "flat top" distortion of the line shape (see Fig. 7). The simple Lorentz and Fano fits of saturated transitions show broader lines and an increased magnitude of QI shifts. To illustrate this effect and obtain numbers that could be used to estimate the size of the QI shift, we compute line shapes in two regimes: high power (HP), when the power of the rf wave corresponds to the estimation of power in the experiment, and low power (LP), when the power is decreased by factor 100 .

The result of our calculation in Table II shows that the QI shift in the worst case, i.e., for the unrealistic scenario of only $M=0$ annihilation detection, is $-618 \mathrm{kHz}$ for a Lorentz fit and $+170 \mathrm{kHz}$ for a Fano fit. The more realistic weighting of 0.74:1:0.74 shows $-59 \mathrm{kHz}$ for a Lorentz fit and $+17 \mathrm{kHz}$ shift for a Fano fit. These results exclude the possibility that the line-shape asymmetry observed in experiment [6] is caused by QI effects. However, it is evident that any improved experiments that are able to obtain uncertainties better than $100 \mathrm{kHz}$ will require more careful studies of QI effects.

Using Fano fits eliminates the QI shift in the low-power regime since the Fano profile approximates the distortion caused by QI. The last column in Table II illustrates this effect. However, using Fano fits for noisy data processing worsens the statistical uncertainty. Therefore, this should be avoided if the uncertainty of the QI shift is smaller than the additional uncertainty of the Fano fit. Another way to eliminate a QI shift is to design a detection scheme with the weights of Zeeman components close to 1:1:1 (see Table II). This may be achieved by directly observing the surviving $2{ }^{3} S_{1}$ population using a microchannel plate detector, for example.

\section{REALISTIC MICROWAVE RADIATION FIELDS}

Reflection of the rf wave that can occur in the experiment [6] may cause line distortions. To show how this may affect the line shape, we first consider a simple model of a reflection from a single point on the $x$ axis. In the framework of a simple model, we show that the reflection may cause asymmetry. We also show that there exist regimes in which the line is strongly shifted without large asymmetry, which present difficulties for data interpretation. After that we consider a more realistic model of the rf field computed from FEM simulation.

\section{A. Simple reflection model}

One of the candidates for the line-shape distortion related to the experiment is a systematic dependence of the rf electricfield amplitude $E_{0}$ on the rf frequency $\omega$. From the KramersHeisenberg formula (14) we see that the transition probability is proportional to the square of electric-field amplitude $R(i \rightarrow$ $f) \sim E_{0}(\omega)^{2}$. Therefore, it is clear that, if the electric field depends on $\omega$, line-shape distortions may occur.

A simple illustration of this effect can be obtained by assuming that a small fraction of the rf power in the waveguide is reflected after some distance $L$ from the interaction region. We can compute an rf electric field affecting an atom at position $x=0$, combining $\vec{E}_{+}$and $\vec{E}_{-}$from equations with a corresponding phase delay and amplitude reflection coefficient $q$ :

$$
\begin{aligned}
E_{z} & \sim E_{0}\left[\cos \left(\omega t+k_{x} x\right)+q \cos \left(\omega t-k_{x} x-2 k_{x} L\right)\right] \\
& =E_{S W}(\omega, x) \cos \left(\omega t+k_{x} x+\phi_{S W}\right),
\end{aligned}
$$




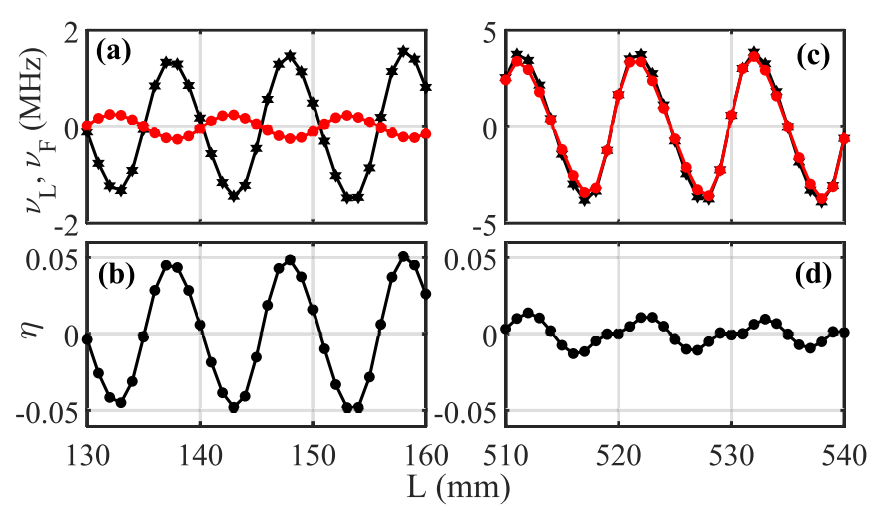

FIG. 10. Centers of Lorentz (hexagons) and Fano (circles) fits [(a) and (c)] and the asymmetry parameter $\eta$ of the Fano fits [(b) and (d)] computed for the $v_{0}$ line vs distance to the reflection point $L$. The amplitude reflection coefficient $q=0.1$ corresponds to $1 \%$ of reflected power. In the small- $L$ regime [(a) and (b)], the difference between Lorentz and Fano fits can be used to characterize the quality of the fits. In a regime of large $L[$ (c) and (d)], the Fano and Lorentz fits give almost equally wrong results, and the difference between them cannot be used for uncertainty estimation. The parameter $\eta$ in the fit function (7) characterizes the line asymmetry and grows as the Fano and Lorentz fit centers diverge from each other.

where $E_{S W}(\omega, x)$ and $\phi_{S W}$ are the amplitude and phase of the rf field in the standing wave, respectively, observed by an atom in a point with coordinate $x$, where

$$
\begin{gathered}
E_{S W}(\omega, x)=E_{0} \sqrt{1+q^{2}+2 q \cos \left[2 k_{x}(L+x)\right]}, \\
\phi_{S W}(\omega, x)=\arcsin \frac{q \sin \left[2 k_{x}(L+x)\right]}{\sqrt{1+q^{2}+2 q \cos \left[2 k_{x}(L+x)\right]}} .
\end{gathered}
$$

The field variations described by Eq. (26) cause a distortion of the line shape, which can easily be included in the numerical model. To study this effect we simulated line shapes varying parameter $L$, using a magnetic field $B=0$, reflection coefficient $q=0.1$, and using equal Zeeman component weights. The centroid values obtained from Lorentz and Fano fits to the obtained data are shown in Fig. 10 for the $v_{0}$ transition as a function of $L$.

As shown in Fig. 10, we observe different effects for small and large values of $L$. If $L$ is small (on the order of several wavelengths), the distortion causes vastly different shifts of the Lorentz and Fano fit centers. Since we use a difference $\delta v=v_{L}-v_{F}$ as a signature of the line asymmetry, we would consider the lines as asymmetric. However, for these lines, we can use $\delta v$ as an estimator of experimental uncertainty.

In contrast, for the large values of $L$ (order of magnitude $\left.\lambda \omega_{0} / \Gamma\right)$, the Lorentz and Fano fits exhibit similarly varying results. Our qualitative estimator of asymmetry $\eta$ shows that lines are much more symmetric than in the regime of small $L$. The difference $\delta v$ is an order of magnitude smaller than the deviation of the fit centers from zero detunings, so the $\delta v$ cannot be used to characterize the uncertainty. In the actual experiment, the reflections can happen anywhere, so the $\delta v$ cannot be used as a reliable uncertainty estimator.

\section{B. Real microwave field simulations}

In the real experimental apparatus, reflections of the $\mathrm{rf}$ waves can happen everywhere. Therefore, a simplified model of the type described above is unlikely to provide an adequate description of the experiment. However, we can simulate the line shape by assuming that the rf field outside the waveguide does not affect Ps atoms, but that the actual field distributions in the surrounding space do have some effect on the fields inside the waveguide. Our approach is the following: first, we numerically compute the real distribution of rf fields inside the vacuum chamber, including the waveguide, using the finite element method. Then we use the results of these simulations to obtain the resulting parameters of the field inside of the waveguide, exploiting the fact that only the $\mathrm{TE}_{10}$ mode can be propagated within the guide. The modified waveguide fields may then be included in the master-equation code to compute the line shape.

\section{Description of the microwave field simulations}

Microwave radiation fields in the full experimental geometry were simulated using CST studio suite software [40]. The layout of the simulation matches the waveguide and chamber setup of [6], with a six way chamber containing either a WR-112, WR-75, or WR-51 waveguide, with the dimensions of each respective waveguide given in Table I, and UV windows to allow the laser light to enter the chamber. The waveguide port models a wire with 50 Ohm impedance. The individual materials present were modeled using the material library so as to emulate the aluminum waveguide, steel chamber, and fused silica windows. CST Studio Suite evaluates 3D electromagnetic fields using a finite element method. The software allows for calculation of the phase and magnitude of the EM field through use of the domain frequency solver. The frequencies simulated match the experimental frequencies sampled from Ref. [6]. An example of the rf field results is shown in Fig. 11. In this case the field was calculated including a WR-112 waveguide in the vacuum at a microwave frequency of $8.624 \mathrm{GHz}$.

The direct calculation of the rf field inside the vacuum chamber shows a strong dependence of the electric field in the waveguide center on the rf frequency. The vacuum chamber acts as an rf cavity with a complicated shape and mode structure, making a quasirandom $E(\omega)$ profile. The effective rf electric fields in the centers of the waveguides are shown in Fig. 12. The rf field amplitude within the frequency scan range varies by up to a factor of 6 , although this may result from underestimating rf absorption in the numerical model.

\section{Waveguide field computation from microwave simulation results}

The Ps velocity distributions (see Fig. 2) can be used to estimate the region of Ps propagation inside the waveguide. According to the experiment [6], the characteristic velocities of Ps atoms are of 20 and $100 \mathrm{~km} / \mathrm{s}$ along $z$ and $x$ axes, respectively, while the atom creation area has approximately $3 \mathrm{~mm}$ in diameter. Thus the Ps atom distribution area has no more than $11 \mathrm{~mm}$ length along the $x$ direction. To obtain the parameters of the rf waves in this region we extract the amplitude of the $z$ component of the electric field along the line with coordinates $z=6+b / 2 \mathrm{~mm}$ and $y=0 \mathrm{~mm}$ corresponding 


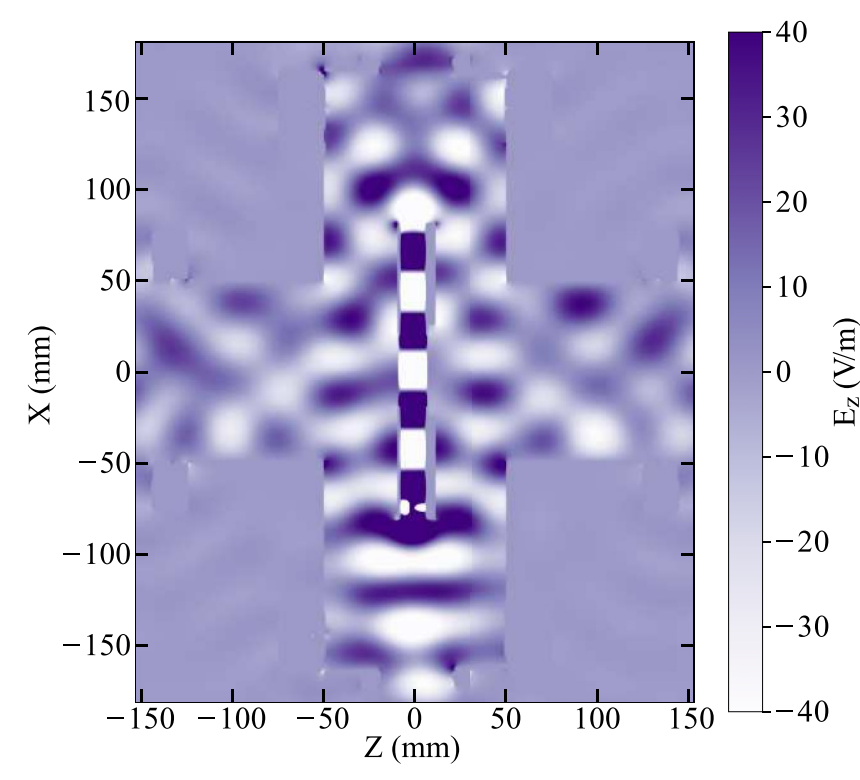

FIG. 11. CST Studio Suite simulation results for the rf field, for a WR-112 waveguide at frequency $8.624 \mathrm{GHz}$ and phase $=0$. This figure demonstrates the $\mathrm{TE}_{10}$ mode inside the waveguide, as well as reflections from the chamber geometry. The field outside the waveguide is 100 times weaker than the field inside the waveguide; however, the peak waveguide field is not shown on the figure for clarity.

to the center of the waveguide (see Fig. 3) and in the range $-13 \mathrm{~mm} \leqslant x \leqslant 13 \mathrm{~mm}$. The amplitude of the field on this line was fitted with function (26) and the parameters $q, E_{0}$, and $L$ were obtained. For better fit qualities the wavelength is considered also as varying parameter. The fitting results show that the standing-wave model of the field inside the waveguide gives an adequate description of the field, which approximates FEM simulation results in the region of Ps atom propagation with inaccuracy less than $2 \%$. The obtained effective amplitude $E_{\text {eff }}(\omega)=E_{\mathrm{SW}}(\omega, x=0)$ for all transitions is shown in Fig. 12. The fit results for the standing waves for different frequencies were cross-checked via different MATLAB and Wolfram Mathematica scripts.

\section{Line shape}

We modified the master equation code to use external data for the rf field instead of constant values of $E_{0}, L$, and $q$. The results of the computation made by this code for all three transitions are summarized in Table III. The line shapes of all transitions are also shown in Fig. 12, along with the effective field dependence on frequency detuning. The computation

TABLE III. Shifts for Lorentz and Fano fits of the results, obtained after including FEM simulated field into the model. The lines were calculated for single atom trajectory with $v_{z}=100 \mathrm{~km} / \mathrm{s}$, $v_{x}=0 \mathrm{~km} / \mathrm{s}$, and zero magnetic field.

\begin{tabular}{lccc}
\hline \hline Waveguide & Line & $v_{L}(\mathrm{MHz})$ & $v_{F}(\mathrm{MHz})$ \\
\hline WR-51 & $v_{0}$ & -3.55 & -2.38 \\
WR-75 & $v_{1}$ & -5.51 & -11.12 \\
WR-112 & $v_{2}$ & -2.34 & +1.80 \\
\hline \hline
\end{tabular}
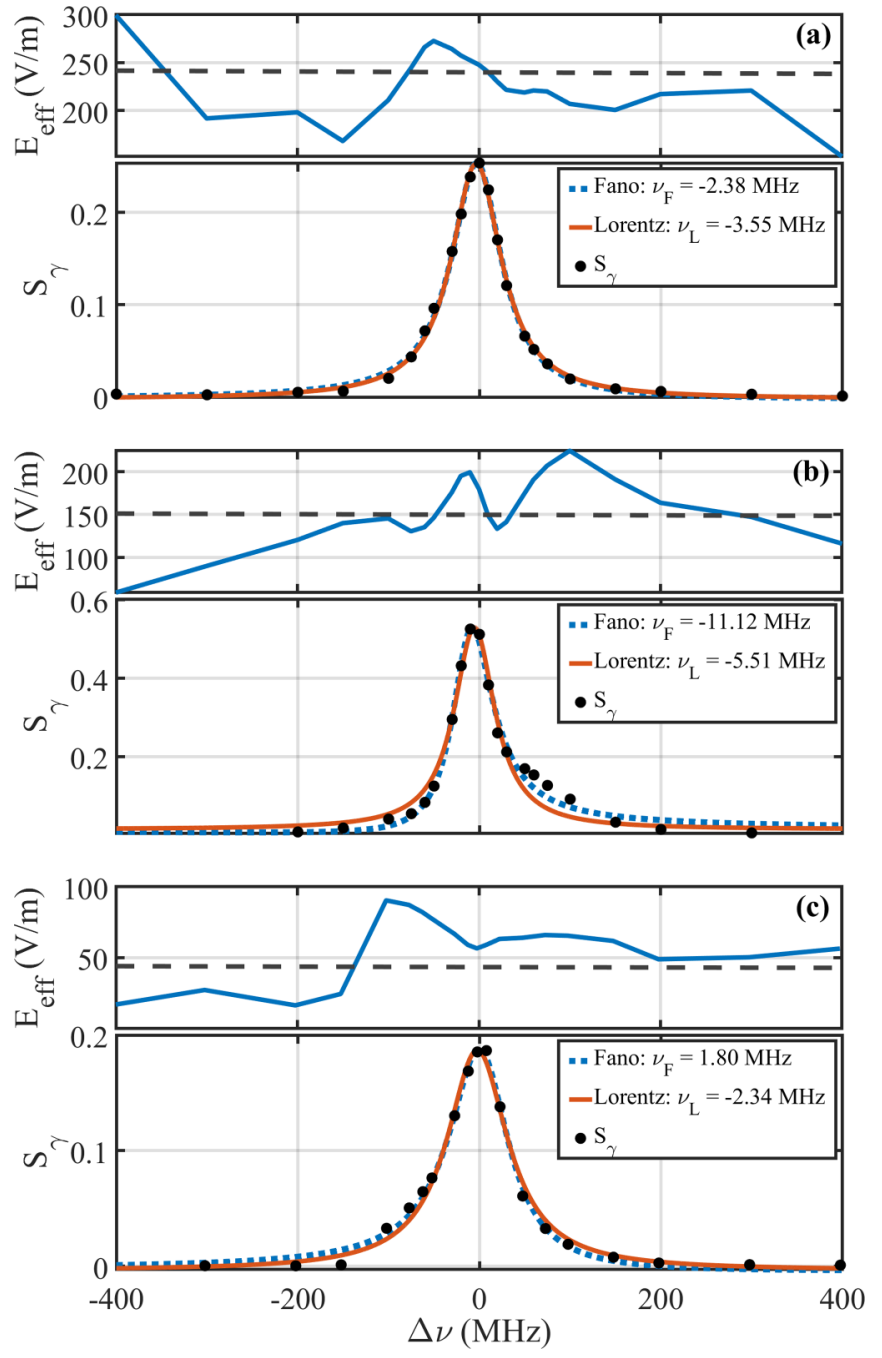

FIG. 12. Effective rf electric-field amplitudes $E_{\text {eff }}$ for $x=0$ in the waveguide, obtained from FEM simulations (solid lines in top sections of each panel) and transition line shapes computed with this field (bottom sections in each panel) with Lorentz and Fano fits for all transitions [ (a), (b), (c) for $v_{0}, v_{1}, v_{2}$, respectively]. The dashed lines in the top figures represent the ideal electric-field amplitudes without reflection calculated with Eq. (8) for each waveguide. Large fluctuations of simulated rf electric field are caused by multiple reflections inside the vacuum chamber. Those fluctuations lead to the uncontrollable behavior of the line shape. The shifts of the Lorentz fits (solid line bottom) and Fano fit (dotted line bottom) for different $J$ are shown.

was done for magnetic field $B=0$ and single velocity $v_{z}=$ $100 \mathrm{~km} / \mathrm{s}$. The $v_{1}$ line shows a large shift of at least $-6 \mathrm{MHz}$, while other transitions show smaller shifts of several $\mathrm{MHz}$ for the Lorentz fitting function.

\section{Uncertainty of the experiment}

The results of the field simulations show that the quasirandom modulation of the rf field amplitude causes line distortions which can mimic the results obtained in experiments. The lines may demonstrate asymmetry (discrepancy between Lorentz and Fano fits) or no asymmetry with a random shift. The root mean square of Lorentz fit center shifts obtained in the simulations is around $4 \mathrm{MHz}$. This result can 
be used as a conservative estimation of the uncertainty of the experiment caused by the rf field reflection.

Additional elements (cables, targets, and holders) not included in the simulation but placed in the vacuum chamber in the actual experiment could change the rf mode structure of the vacuum chamber. Due to that, the simulation cannot be used for a direct correction of the experimental results. However, those elements could possibly absorb the rf power, decreasing the quality factor of the vacuum chamber as an rf cavity. Due to that, the field simulations could overestimate the quasirandom variations of rf amplitude.

\section{OTHER SYSTEMATIC EFFECTS}

Using the master-equation model it is also possible to study other perturbations that could affect Ps fine-structure measurements. We found that the considered effects are negligible at a level of accuracy on the order of $200 \mathrm{kHz}$. Although these effects are not presently limiting measurements we briefly describe them here because they may become important in future measurements.

To estimate how a particular perturbation shifts the line, we computed perturbed and unperturbed line shapes for all three transitions' fine structure. We use a difference between perturbed and unperturbed Lorentz and Fano fit centers to estimate the magnitude of the shift. The calculation of line shape was completed using a single trajectory with velocity $100 \mathrm{~km} / \mathrm{s}$, magnetic field $32 \mathrm{G}$, and equal distribution of the Zeeman components' weights. The following effects were considered.

a. Imperfect magnetic-field alignment. We introduce an imperfect alignment by tilting the magnetic-field direction by $10^{\circ}$ from its ideal orientation. The simulation shows that this changes the position of the fit centers for $v_{0}, v_{1}$, and $v_{2}$ components by approximately $-10,-29$, and $+32 \mathrm{kHz}$ for both for Lorentz and Fano fits.

b. Polarization of positron beam. Because it is derived from the beta decay of a radioactive source, the positron beam used in the experiments is partially spin polarized [41]. Previous experiments have indicated that the beam polarization is $\approx 0.3$ [42], meaning that the Ps atoms will also be partially spin polarized [43]. This polarization can be modeled via unequal populations of $2{ }^{3} S_{1}(M=-1)$ and $2{ }^{3} S_{1}(M=+1)$ states at the beginning of evolution. To match the experimentally measured polarization, the initial populations of corresponding states were set to $14 / 60$ and $26 / 60$. We found that the line does not show any asymmetry and the difference between the Lorentz fit central frequency for polarized and unpolarized beams does not exceed $2 \mathrm{kHz}$ for all fine-structure components.

c. Tilted polarization axis of rf field. Misalignment of the polarization of the electric field is almost impossible because it could happen only if the waveguide is mechanically tilted. However, we checked what happens if the electric field of the rf wave polarization axis is $10^{\circ}$ off from its normal direction along the $z$ axis. The simulations indicate that the lines are shifted by approximately $-10,-29$, and $+32 \mathrm{kHz}$ for both the Lorentz and Fano fits.

d. Imperfect polarization near the meshes. In the actual experiment, atoms enter and exit the waveguide through the meshes in its upper and lower walls. The electric-field vector near the meshes may have random variations due to the presence of wires. To mimic this effect we introduce in the model a tilt of the $\mathrm{rf}$ wave electric field by angle $\theta(z)=$ $\theta_{0}\left[\left(z-z_{a}\right) / b\right]^{4}$, where $\theta_{0}=45^{\circ}, z_{a}$ is a $z$ coordinate of the waveguide wall, and $b$ is a height of the waveguide. The simulation shows that the lines are shifted approximately by $-1,-2$, and $+3 \mathrm{kHz}$ for both the Lorentz and Fano fits.

e. High harmonics in $r f$ wave. We model an effect for the second and third harmonic using replacement $\cos (\omega t)$ by $\cos (\omega t)+0.5 \cos (N \omega t)$, where $N=2,3$. The Lorentz fits for the second harmonic show the shift of the line center by -1.7 , -1.2 , and $+1.0 \mathrm{kHz}$; corresponding shifts for Fano fits are $-1.7,-1.2$, and $-1.5 \mathrm{kHz}$. The shifts for third harmonic distortion are also small, not exceeding $1 \mathrm{kHz}$ for both Lorentz and Fano fits of all transitions.

\section{CONCLUSIONS}

We have described a numerical model related to positronium fine-structure spectroscopy experiments [6]. The model is based on the formalism of a master equation in the Lindblad form, and allows us to include a variety of experimental effects and investigate subsequent systematic shifts. We calculated quantum interference effects and show conclusively that they are negligible at the current level of experimental precision.

A finite element simulation of the microwave radiation, including reflections from the surrounding vacuum chamber, was performed. We found that reflections of $\mathrm{rf}$ waves may generate fields inside the waveguides that are able to cause distortions of the resulting line shapes, arising from frequency-dependent variations in the radiation field. Depending on the details of the reflections, this effect can lead to asymmetric line shapes, and may even shift the line without visible asymmetry (as characterized by the difference between Fano and Lorentz fits; see Fig. 10). We also reproduce qualitatively the observations [6] of both positive and negative shifts. The observed Fano-Lorentz difference is on the order of a few $\mathrm{MHz}$, which is comparable to those predicted by the simulations. The root mean square of Lorentz fit center shifts for simulated line profiles is about $4 \mathrm{MHz}$, which can be used as an estimation of uncertainty caused by rf wave reflection.

An improvement of the experimental uncertainty requires solving the problem of frequency-dependent $\mathrm{rf}$ wave amplitudes. We expect that this can be achieved by redesigning the waveguide and vacuum chamber elements so as to eliminate reflections, or by constructing an active monitoring and control system. In that case, the new methods employed [6] should make it possible to achieve measurements of the Ps $n=2$ fine structure with a precision at the $100 \mathrm{kHz}$ level and bring the experimental uncertainties in line with those of theory.

\section{ACKNOWLEDGMENTS}

This work was funded in part by the EPSRC under Grant No. EP/R006474/1. We gratefully acknowledge helpful discussions with P. Crivelli, S. D. Hogan, and R. Pohl. We would like to thank MPCDF Garching for computation time. 
[1] J. A. Wheeler, Polyelectrons, Ann. (NY) Acad. Sci. 48, 219 (1946).

[2] S. G Karshenboim, Precision study of positronium: Testing bound state QED theory, Int. J. Mod. Phys. A 19, 3879 (2004).

[3] D. B. Cassidy, Experimental progress in positronium laser physics, Eur. Phys. J. D 72, 53 (2018).

[4] A. Czarnecki, K. Melnikov, and A. Yelkhovsky, Positronium $S$-state spectrum: Analytic results at $o\left(m \alpha^{6}\right)$, Phys. Rev. A 59, 4316 (1999).

[5] L. Gurung, T. J. Babij, S. D. Hogan, and D. B. Cassidy, Precision Microwave Spectroscopy of the Positronium $n=2$ Fine Structure, Phys. Rev. Lett. 125, 073002 (2020).

[6] L. Gurung, T. J. Babij, J. Pérez-Ríos, S. D. Hogan, and D. B. Cassidy, Observation of asymmetric line shapes in precision microwave spectroscopy of the positronium $2^{3} S_{1} \rightarrow 2^{3} P_{J}(J=$ 1, 2) fine-structure intervals, Phys. Rev. A 103, 042805 (2021).

[7] A. P. Mills, Jr., S. Berko, and K. F. Canter, Fine-Structure Measurement in the First Excited State of Positronium, Phys. Rev. Lett. 34, 1541 (1975).

[8] S. Hatamian, R. S. Conti, and A. Rich, Measurements of the $2^{3} S_{1}-2^{3} P_{J}(J=0,1,2)$ Fine-Structure Splittings in Positronium, Phys. Rev. Lett. 58, 1833 (1987).

[9] D. Hagena, R. Ley, D. Weil, G. Werth, W. Arnold, and H. Schneider, Precise Measurement of $n=2$ Positronium FineStructure Intervals, Phys. Rev. Lett. 71, 2887 (1993).

[10] J. R. Danielson, D. H. E. Dubin, R. G. Greaves, and C. M. Surko, Plasma and trap-based techniques for science with positrons, Rev. Mod. Phys. 87, 247 (2015).

[11] M. Horbatsch and E. A. Hessels, Shifts from a distant neighboring resonance, Phys. Rev. A 82, 052519 (2010).

[12] T. Udem, L. Maisenbacher, A. Matveev, V. Andreev, A. Grinin, A. Beyer, N. Kolachevsky, R. Pohl, D. C. Yost, and T. W. Hänsch, Quantum interference line shifts of broad dipoleallowed transitions, Ann. Phys. (Leipzig) 531, 1900044 (2019).

[13] A. Beyer, L. Maisenbacher, A. Matveev, R. Pohl, K. Khabarova, A. Grinin, T. Lamour, D. C. Yost, T. W. Hänsch, N. Kolachevsky, and T. Udem, The Rydberg constant and proton size from atomic hydrogen, Science 358, 79 (2017).

[14] N. Bezginov, T. Valdez, M. Horbatsch, A. Marsman, A. C. Vutha, and E. A. Hessels, A measurement of the atomic hydrogen Lamb shift and the proton charge radius, Science $\mathbf{3 6 5}$, 1007 (2019).

[15] G. Lindblad, On the generators of quantum dynamical semigroups, Commun. Math. Phys. 48, 119 (1976).

[16] A. M. Alonso, S. D. Hogan, and D. B. Cassidy, Production of $2^{3} S_{1}$ positronium atoms by single-photon excitation in an electric field, Phys. Rev. A 95, 033408 (2017).

[17] L. Liszkay, C. Corbel, P. Perez, P. Desgardin, M. F. Barthe, T. Ohdaira, R. Suzuki, P. Crivelli, U. Gendotti, A. Rubbia, M. Etienne, and A. Walcarius, Positronium reemission yield from mesostructured silica films, Appl. Phys. Lett. 92, 063114 (2008).

[18] B. S. Cooper, A. M. Alonso, A. Deller, T. E. Wall, and D. B. Cassidy, A trap-based pulsed positron beam optimised for positronium laser spectroscopy, Rev. Sci. Instrum. 86, 103101 (2015).

[19] D. B. Cassidy, S. H. M. Deng, R. G. Greaves, T. Maruo, N. Nishiyama, J. B. Snyder, H. K. M. Tanaka, and A. P. Mills, Jr., Experiments with a High-Density Positronium Gas, Phys. Rev. Lett. 95, 195006 (2005).
[20] D. B. Cassidy, P. Crivelli, T. H. Hisakado, L. Liszkay, V. E. Meligne, P. Perez, H. W. K. Tom, and A. P. Mills, Jr., Positronium cooling in porous silica measured via Doppler spectroscopy, Phys. Rev. A 81, 012715 (2010).

[21] M. H. Rayment, L. Gurung, R. E. Sheldon, S. D. Hogan, and D. B. Cassidy, Multiring electrostatic guide for Rydberg positronium, Phys. Rev. A 100, 013410 (2019).

[22] Y. Nagashima, Y. Morinaka, T. Kurihara, Y. Nagai, T. Hyodo, T. Shidara, and K. Nakahara, Origins of positronium emitted from $\mathrm{SiO}_{2}$, Phys. Rev. B 58, 12676 (1998).

[23] P. Crivelli, U. Gendotti, A. Rubbia, L. Liszkay, P. Perez, and C. Corbel, Measurement of the orthopositronium confinement energy in mesoporous thin films, Phys. Rev. A 81, 052703 (2010).

[24] B. S. Cooper, A. M. Alonso, A. Deller, L. Liszkay, and D. B. Cassidy, Positronium production in cryogenic environments, Phys. Rev. B 93, 125305 (2016).

[25] D. B. Cassidy, S. H. M. Deng, H. K. M. Tanaka, and A. P. Mills, Jr., Single shot positron annihilation lifetime spectroscopy, Appl. Phys. Lett. 88, 194105 (2006).

[26] U. Fano, Effects of configuration interaction on intensities and phase shifts, Phys. Rev. 124, 1866 (1961).

[27] U. D. Jentschura and P. J. Mohr, Nonresonant effects in one- and two-photon transitions, Can. J. Phys. 80, 633 (2002).

[28] C.J. Foot, Atomic Physics, Oxford Master Series in Physics (Oxford University Press, Oxford, 2005).

[29] Z. Ficek and S. Swain, Quantum Interference and Coherence (Springer-Verlag New York, 2005).

[30] G. S. Agarwal, Quantum Statistical Theories of Spontaneous Emission and their Relation to Other Approaches (SpringerVerlag, Berlin, 1974).

[31] A. Marsman, M. Horbatsch, and E. A. Hessels, Interference between two resonant transitions with distinct initial and final states connected by radiative decay, Phys. Rev. A 96, 062111 (2017).

[32] A. I. Alekseev, Two-photon annihilation of positronium in the P-state, ZhETF 34, 1195 (1958) [Sov. Phys. JETP 7, 826 (1958)].

[33] A. I. Alekseev, Three-photon annihilation of positronium in the P-state, ZhETF 36, 1839 (1959) [Sov. Phys. JETP 9, 1312 (1959)].

[34] Ginac c++ library, https://www.ginac.de.

[35] W. H. Press, S. A. Teukolsky, W. T. Vetterling, and B. P. Flannery, Numerical Recipes: The Art of Scientific Computing, 3rd ed. (Cambridge University Press, Cambridge, UK, 2007).

[36] R. C. Brown, S. Wu, J. V. Porto, C. J. Sansonetti, C. E. Simien, S. M. Brewer, J. N. Tan, and J. D. Gillaspy, Quantum interference and light polarization effects in unresolvable atomic lines: Application to a precise measurement of the ${ }^{6,7} \mathrm{Li} D_{2}$ lines, Phys. Rev. A 87, 032504 (2013).

[37] D. C. Yost, A. Matveev, E. Peters, A. Beyer, T. W. Hänsch, and T. Udem, Quantum interference in two-photon frequency-comb spectroscopy, Phys. Rev. A 90, 012512 (2014).

[38] H. Fleurbaey, F. Biraben, L. Julien, J.-P. Karr, and F. Nez, Cross-damping effects in $1 s-3 s$ spectroscopy of hydrogen and deuterium, Phys. Rev. A 95, 052503 (2017) 
[39] R. M. Drisko, Spin and polarization effects in the annihilation of triplet positronium, Phys. Rev. 102, 1542 (1956).

[40] Cst studio suite, dassault systemes, https://www.3ds.com/ products-services/simulia/products/cst-studio-suite/.

[41] P. G. Coleman, Positron Beams and Their Applications, 1st ed. (World Scientific Publishing Co. Singapore, 2000).
[42] D. B. Cassidy, V. E. Meligne, and A. P. Mills, Jr., Production of a Fully Spin-Polarized Ensemble of Positronium Atoms, Phys. Rev. Lett. 104, 173401 (2010).

[43] B. K. Arbic, S. Hatamian, M. Skalsey, J. Van House, and W. Zheng, Angular-correlation test of CPT in polarized positronium, Phys. Rev. A 37, 3189 (1988). 